\title{
Improving updating rules in multiplicative algorithms for computing $D$-optimal designs
}

\author{
Holger Dette \\ Andrey Pepelyshev \\ Ruhr-Universität Bochum \\ Fakultät für Mathematik \\ 44780 Bochum, Germany \\ e-mail: holger.dette@rub.de \\ FAX: +492343214 559 \\ St. Petersburg State University \\ Department of Mathematics \\ St. Petersburg \\ Russia \\ email: andrey@ap7236.spb.edu \\ Anatoly Zhigljavsky \\ Cardiff University \\ School of Mathematics \\ Cardiff CF24 4AG, UK \\ FAX: +44 (0) 2920874199 \\ email: ZhigljavskyAA@cf.ac.uk
}

August 16, 2007

\begin{abstract}
In this paper we discuss a class of multiplicative algorithms for computing $D$-optimal designs for regression models on a finite design space. We prove a monotonicity result for a sequence of determinants obtained by the iterations, and as a consequence the procedure yields a sequence of designs converging to the $D$-optimal design. The class of algorithms is indexed by a real parameter and contains two algorithms considered by Titterington $(1976,1978)$ as special cases. We provide numerical results demonstrating the efficiency of the proposed methods and discuss several extensions to other optimality criteria.
\end{abstract}

Keywords and phrases: $D$-optimal design, finite design space, multiplicative algorithm, minimal covering ellipsoid 


\section{Introduction}

Consider the common linear regression model

$$
y=\theta^{T} u+\varepsilon,
$$

where $\theta=\left(\theta_{0}, \ldots, \theta_{m-1}\right)^{T} \in \mathbb{R}^{m}$ is a vector of unknown parameters, $u$ denotes the vector of explanatory variables and $\varepsilon$ is a random error. We assume that $\mathcal{U}=\left\{u_{1}, \ldots, u_{n}\right\}$ $\subset \mathbb{R}^{m}$ is a finite design space (a generalization to arbitrary design spaces is straightforward, see Remark 4 in Section 2) and that different observations are uncorrelated; the mean and variance of the errors are 0 and $\sigma^{2}>0$, respectively. Following Kiefer (1974) we call any probability measure $\xi$ on $\mathcal{U}$ a design. If $N$ observations can be taken and the design $\xi$ puts masses $w_{1}, \ldots, w_{n}$ at the points $u_{1}, \ldots, u_{n}$, then a rounding procedure is applied to obtain integers $n_{i} \approx w_{i} N$ with $\sum_{j=1}^{n} n_{i}=N$, and the experimenter takes approximately $n_{i}$ observations at each $u_{i}(i=1, \ldots, n)$ [see Pukelsheim and Rieder (1992) for more details and some references]. For a design $\xi$, the information matrix in the model (1) is defined by

$$
M(\xi)=\sum_{i=1}^{n} w_{i} u_{i} u_{i}^{T},
$$

and approximately proportional to the covariance matrix of the least squares estimate for the parameter $\theta$. An optimal design maximizes an appropriate function of the information matrix [see e.g. Silvey (1980) or Pukelsheim (1993)]. Numerous optimality criteria have been proposed in the literature to discriminate between competing designs. In the present paper we mainly consider the $D$-optimality criterion, which determines the design $\xi^{*}$ such that the determinant $|M(\xi)|$ is maximal. Such a design minimizes the volume of the ellipsoid of concentration for the vector $\theta$ of unknown parameters. In most cases of practical interest, $D$-optimal designs have to be determined numerically and several algorithms have been proposed in the literature for computing $D$-optimal designs [see e.g. Fedorov (1972), Wynn (1972), Silvey (1980), Pazman (1986) or the recent papers of Harman and Pronzato (2007), Mandal and Torsney (2006)].

In the present paper we concentrate on a class of multiplicative algorithms for computing $D$-optimal designs, which is indexed by real parameters, say $\beta_{r}$. For two special choices of the indexes $\beta_{r}$ the algorithms are related to two algorithms proposed by Titterington $(1976,1978)$ and Silvey, Titterington and Torsney (1978). In Section 2 we prove that the sequence of determinants of the corresponding designs is nondecreasing, and as a consequence the sequence of calculated designs converges to the $D$-optimal design. The monotonicity result uses particular thresholds in each step of the iterations, and some bounds for these thresholds are derived in Section 3. Some results of numerical comparisons are given in Section 4. In Section 5, some applications of multiplicative algorithms to the construction of optimal designs with respect to other optimality criteria are considered. 


\section{A class of multiplicative algorithms for calculat- ing $D$-optimal designs}

Let $w_{1}^{(0)}, \ldots, w_{n}^{(0)}$ denote a set of given initial weights for the design points $u_{1}, \ldots, u_{n}$, which defines the initial design $\xi^{(0)}$. For a design $\xi$ with weights $w_{i}$ at the points $u_{i}$, we define

$$
d\left(u_{i}, \xi\right)=\frac{\partial}{\partial w_{i}} \log \operatorname{det} M(\xi)=u_{i}^{T} M^{-1}(\xi) u_{i} \quad(i=1, \ldots, n) .
$$

We study the class of multiplicative algorithms for calculating $D$-optimal designs which is defined recursively by updating the weights in each step as follows:

$$
w_{i}^{(r+1)}=w_{i}^{(r)} \frac{d\left(u_{i}, \xi^{(r)}\right)-\beta_{r}}{m-\beta_{r}}, \quad \beta_{r} \in \mathbb{R} .
$$

Here $r=0,1, \ldots$ is the iteration number and $\beta_{r}(r=0,1, \ldots)$ are real-valued parameters that can vary in each iteration. For an initial design $\xi^{(0)}$, the iterations (2) produce a sequence of designs $\left\{\xi^{(r)}\right\}_{r=0,1, \ldots}$.

The choices of constants $\beta_{r}=0$ and $\beta_{r}=1$ in the algorithm (2) give two procedures considered by Titterington $(1976,1978)$, who discussed a special case of model (1), where the first component of the vector $u$ in the regression model (1) is constant, i.e. $u=\left(1, v^{T}\right)^{T}, v \in \mathbb{R}^{m-1}$. This author established that in the case $\beta_{r}=0$ the algorithm (2) yields a sequence of determinants $\left\{\operatorname{det} M\left(\xi^{(r)}\right)\right\}$, which is nondecreasing and converges to the $D$-optimal design. A more detailed proof of the same result is given by Pazman (1986). Note that there is an alternative proof of J.F.C. Kingman (unpublished). Based on numerical experiments Silvey, Titterington and Torsney (1978) and Titterington $(1976,1978)$ noticed that for $\beta_{r}=1$ the algorithm converges substantially faster than for $\beta_{r}=0$ and conjectured that the sequence of determinants for algorithm (2) with $\beta_{r}=1$ is also nondecreasing. There is a vast literature where the rates of convergence of the algorithm (2) with $\beta_{r} \in\{0,1\}$ and related algorithms are numerically studied and the monotonicity conjecture for the case of $\beta_{r}=1$ is numerically verified, see e.g. Pázman (1986); Pukelsheim and Torsney (1991); Torsney and Mandal (2001); Pronzato (2003); Harman and Pronzato (2007); Torsney (2007) and Pronzato et al. (2000), p.155.

Theorem 1 below does not give a proof of the monotonicity conjecture in the case $\beta_{r}=1$, but establishes the monotonicity of the determinants $\left\{\operatorname{det} M\left(\xi^{(r)}\right)\right\}$ for certain positive values of $\beta_{r}$. For a precise formulation of the statement, we define for the design $\xi^{(r)}$ in the $r$-th step the quantity

$$
\beta^{(r)}=\min _{u_{i} \in \mathcal{U}} d\left(u_{i}, \xi^{(r)}\right)
$$

which turns out to be essential in the following discussion. Note that we must always have $\beta_{r} \leq \beta^{(r)}$ as otherwise at least one weight in the updated design $\xi^{(r+1)}$ becomes negative. 
Theorem 1. Let $\left\{\xi^{(r)}\right\}_{r=0,1, \ldots}$ be a sequence of designs obtained by the recurrent formula (2), where in the $r$-th step the parameter $\beta_{r}$ is chosen as any number such that

$$
-\infty<\beta_{r} \leq \beta^{(r)} / 2
$$

then the sequence of determinants $\left\{\operatorname{det} M\left(\xi^{(r)}\right)\right\}_{r=0,1, \ldots}$ is nondecreasing.

The proof (generalizing the proof of Pázman (1986) valid for $\beta_{r}=0$ ) is based on several auxiliary lemmas.

Lemma 1. Let $\zeta_{1}, \ldots, \zeta_{m}$ be i.i.d. random variables with values in $\mathcal{U}=\left\{u_{1}, \ldots, u_{n}\right\}$ and distribution $\xi$, and let $\alpha$ be some positive constant. Let also $g: \mathcal{U}^{m} \rightarrow[0, \infty)$ denote a function such that $E\left(g \mid \zeta_{i}\right) \geq 2 \alpha$ a.s. (almost surely), where $g=g\left(\zeta_{1}, \ldots, \zeta_{m}\right)$. Then

$$
E(g)[E(g)-\alpha]^{m} \leq E\left[g \prod_{i=1}^{m}\left(E\left(g \mid \zeta_{i}\right)-\alpha\right)\right]
$$

and the equality is strict unless $E\left(g \mid \zeta_{1}\right)=\ldots=E\left(g \mid \zeta_{m}\right)=E(g)$ a.s.

Lemma 2. Let $h: \mathcal{U}^{m} \rightarrow \mathbb{R}$ be the function defined by

$$
h\left(z_{1}, \ldots, z_{m}\right)=\frac{1}{m !} \operatorname{det}^{2} F\left(z_{1}, \ldots, z_{m}\right),
$$

where $z_{i} \in \mathcal{U}(i=1, \ldots, m)$ and $i$-th column of the matrix $F\left(z_{1}, \ldots, z_{m}\right)$ equals $z_{i}$. Then, for a design with weights $w_{1}, \ldots, w_{n}$ at the points $u_{1}, \ldots, u_{n}$, the determinant of the information matrix $M(\xi)=\sum_{i=1}^{n} w_{i} u_{i} u_{i}^{T}$ can be represented as

$$
\operatorname{det} M(\xi)=E h\left(\zeta_{1}, \ldots, \zeta_{m}\right)=\sum_{i_{1}=1}^{n} \ldots \sum_{i_{m}=1}^{n} w_{i_{1}} \cdots w_{i_{m}} h\left(u_{i_{1}}, \ldots, u_{i_{m}}\right)
$$

where $\zeta_{1}, \ldots, \zeta_{m}$ are i.i.d. random variables with distribution $\xi$.

Lemma 3. For any $\beta \in \mathbb{R}(\beta \neq m)$, any $u \in \mathcal{U}$ and any $j(1 \leq j \leq m)$ we have

$$
\frac{d(u, \xi)-\beta}{m-\beta}=\frac{E h\left(\zeta_{j}^{u}\right)-\alpha}{E h(\zeta)-\alpha}
$$

where $\zeta=\left(\zeta_{1}, \ldots, \zeta_{m}\right), \zeta_{j}^{u}=\left(\zeta_{1}, \ldots, \zeta_{j-1}, u, \zeta_{j+1}, \ldots, \zeta_{m}\right), \alpha=\beta E h(\zeta) / m, h(\cdot)$ is defined in (4) and $\zeta_{1}, \ldots, \zeta_{m}$ are (as in Lemmas 1 and 2) i.i.d. random variables with distribution $\xi$.

Proof of Lemma 1. From the inequality $t-1 \geq \log (t)$ we obtain

$$
\prod_{i=1}^{m} \frac{E\left(g \mid \zeta_{i}\right)-\alpha}{E(g)-\alpha}-1 \geq \sum_{i=1}^{m}\left[\log \left(E\left(g \mid \zeta_{i}\right)-\alpha\right)-\log (E(g)-\alpha)\right] \text { a.s. }
$$


Multiplying both sides by $g$ and taking the expectation we obtain

$$
\begin{aligned}
E\left\{g \prod_{i=1}^{m} \frac{E\left(g \mid \zeta_{i}\right)-\alpha}{E(g)-\alpha}\right\}-E(g) & \\
& \geq \sum_{i=1}^{m}\left[E\left(g \log \left(E\left(g \mid \zeta_{i}\right)-\alpha\right)\right)-E(g) \log (E(g)-\alpha)\right] \\
& =\sum_{i=1}^{m}\left[E\left[E\left(g \mid \zeta_{i}\right) \log \left(E\left(g \mid \zeta_{i}\right)-\alpha\right)\right]-E(g) \log (E(g)-\alpha)\right] \geq 0 .
\end{aligned}
$$

The last inequality follows from Jensen's inequality $E \phi(t) \geq \phi(E(t))$ with the strictly convex function $\phi(t)=t \log (t-\alpha), t \in[2 \alpha, \infty)$.

Proof of Lemma 2. This is a consequence of the Binet-Cauchy formula [see Gantmacher (1959)].

Proof of Lemma 3. By the definition, for any $j(1 \leq j \leq m)$ and $u=u_{i} \in \mathcal{U}$ we have

$$
d(u, \xi)=\frac{\partial \log \operatorname{det} M(\xi)}{\partial w_{i}}=\frac{1}{\operatorname{det} M(\xi)} \frac{\partial \operatorname{det} M(\xi)}{\partial w_{i}}=\frac{m E h\left(\zeta_{j}^{u}\right)}{E h(\zeta)}
$$

where we have used (5). Therefore

$$
\frac{d(u, \xi)}{m}=\frac{E h\left(\zeta_{j}^{u}\right)}{E h(\zeta)}
$$

which yields

$$
\frac{d(u, \xi)-\beta}{m-\beta}=\frac{d(u, \xi) / m-\beta / m}{1-\beta / m}=\frac{E h\left(\zeta_{j}^{u}\right)-\alpha}{E h(\zeta)-\alpha}
$$

where $\alpha=\beta E h(\zeta) / m$.

Proof of Theorem 1. Let $\zeta_{1}, \ldots, \zeta_{m}$ be i.i.d. random variables with distribution $\xi^{(r)}, h=h\left(\zeta_{1}, \ldots, \zeta_{m}\right)$ and $\alpha=\beta_{r} E h / m$. Note that (3) implies $E\left(h \mid \zeta_{i}\right) \geq 2 \alpha$ a.s. This allows us to apply Lemma 1. Applying it along with Lemma 2 with $g=h$ and Lemma 3 (taking into account that $E h\left(\zeta_{j}^{u}\right)=E\left(h(\zeta) \mid \zeta_{j}=u\right)$ ) we have

$$
\operatorname{det} M\left(\xi^{(r+1)}\right)=E\left\{h \prod_{i=1}^{m} \frac{E\left(h \mid \zeta_{i}\right)-\alpha}{E h-\alpha}\right\} \geq E h=\operatorname{det} M\left(\xi^{(r)}\right) .
$$

This proves the monotonicity result.

Remark 1 (sharpness of the main result).

Theorem 1 is sharp in the following sense. If we consider the algorithm (2) with $\beta_{r}=\gamma \beta^{(r)}$ and fixed $\gamma$, then $\gamma^{*}=\frac{1}{2}$ is the largest possible value of $\gamma$ such that 
the algorithm (2) yields a monotonic sequence of determinants $\left\{\operatorname{det} M\left(\xi^{(r)}\right)\right\}$ for any regression model, any $\mathcal{U}$ and any initial design (note that for some models and some initial designs the value of $\gamma$ can be increased, see below).

Indeed, consider the regression model $y=\theta^{T} u+\varepsilon$, where $\mathcal{U}=\left\{u_{0}, u_{1}\right\}=\left\{(1,0)^{T},(1,1)^{T}\right\}$, and let the weights of a two-point design $\xi^{(0)}$ be $w_{0}=\frac{1}{2}+\varepsilon$ and $w_{1}=\frac{1}{2}-\varepsilon$ for some small $\varepsilon>0$. Assume that $\beta_{r}=\left(\frac{1}{2}+\delta\right) \beta^{(r)}$ for some small $\delta>0$. It is easy to compute

$$
\operatorname{det} M\left(\xi^{(1)}\right)-\operatorname{det} M\left(\xi^{(0)}\right)=\frac{8 \varepsilon^{2}(1+2 \varepsilon)}{(1+4 \varepsilon-2 \delta)^{2}}(\varepsilon-\delta)
$$

which is negative if $\delta>\varepsilon$.

Remark 2 (regression model with intercept). Consider the regression model with intercept, where

$$
u=\left(1, v^{T}\right)^{T}, v \in \mathbb{R}^{m-1} .
$$

It is easy to show [see Titterington (1978)] that in this case the function $d\left(u_{i}, \xi\right)$ can be written as $d\left(u_{i}, \xi\right)=d_{c}\left(v_{i}, \xi\right)+1$, where

$$
\begin{gathered}
d_{c}\left(v_{i}, \xi\right)=\left(v_{i}-\bar{v}\right)^{T} M_{c}^{-1}\left(v_{i}-\bar{v}\right), \\
M_{c}=\sum_{i=1}^{n} w_{i}\left(v_{i}-\bar{v}\right)\left(v_{i}-\bar{v}\right)^{T}, \bar{v}=\sum_{i=1}^{n} w_{i} v_{i} .
\end{gathered}
$$

Therefore, $d\left(u_{i}, \xi\right) \geq 1$ for any $u_{i} \in \mathcal{U}$. As a consequence of Theorem 1 , the algorithm in the model with intercept is monotonic for any $\beta_{r} \leq \frac{1}{2}$. Note that for some models and designs $\xi^{(r)}$, the values of $\beta_{r}=\beta^{(r)} / 2$ may be much larger than 1 .

Remark 3 (minimal volume ellipsoids). As demonstrated by many authors [see e.g. Titterington (1975, 1978); Pronzato (2003)] the multiplicative algorithms for finding $D$-optimal designs play an important role in construction of a minimal volume ellipsoid containing a set of points

$$
\mathcal{V}=\left\{v_{1}, \ldots, v_{n}\right\} \in \mathbb{R}^{m-1}
$$

Indeed, let the data set consists of the points (7). Then the minimal covering ellipsoid for the set $\mathcal{V}$ is of the form

$$
\mathcal{E}(\mathcal{V})=\left\{v: d_{c}(v, \xi) \leq m-1\right\}
$$

where $d_{c}(v, \xi)$ is defined in (6). In other terms this ellipsoid has the form

$$
\{u: d(u, \xi) \leq m\}
$$

By the equivalence theory of Kiefer and Wolfowitz (1960) we have that the point $v_{i}$ has a positive weight $w_{i}$ only if it lies on the surface of the ellipsoid $\mathcal{E}(\mathcal{V})$. Thus, the points 
that lie on the surface of the minimal volume ellipsoid can be found from the solution of the $D$-optimal design problem on a finite set. Therefore, the algorithms considered in Theorem 1 can be considered as algorithms of construction of the minimal volume ellipsoids containing given sets of points. As illustrated below on some numerical examples, these algorithms may have much faster convergence than the algorithms considered in Titterington $(1976,1978)$.

Remark 4 (general design spaces). In the case of general design spaces $\mathcal{U}$ and general measures, the algorithm (2) becomes

$$
d \xi^{(r+1)}(u)=\frac{d\left(u, \xi^{(r)}\right)-\beta_{r}}{m-\beta_{r}} d \xi^{(r)}(u), \quad \beta_{r} \in \mathbb{R}, \quad u \in \mathcal{U} .
$$

In particular, if the initial design $\xi^{(0)}$ has a density, then all designs $\xi^{(r)}$ have densities $p_{r}(u)$, that is $d \xi^{(r)}(u)=p_{r}(u) d u$, and the updating formula (8) has the form

$$
p_{r+1}(u)=\frac{d\left(u, \xi^{(r)}\right)-\beta_{r}}{m-\beta_{r}} p_{r}(u), \quad \beta_{r} \in \mathbb{R}, \quad u \in \mathcal{U}
$$

Theorem 1 can be generalized to establish monotonicity of determinants for iterations (8).

\section{A lower bound for the function $d$}

In this section we derive a lower bound for the function $d$. Recall the definition of the Elfving set corresponding to the linear regression model (1), i.e.

$$
\mathcal{G}=\operatorname{conv}\left\{u_{1}, \ldots, u_{n},-u_{1}, \ldots,-u_{n}\right\}
$$

[see Elfving (1952) or Pukelsheim (1993)], where $\operatorname{conv}(A)$ denotes the convex hull of a set $A$. Note that $\mathcal{G}$ is a nonempty convex set containing the origin if there are $m$ linearly independent vectors among $u_{1}, \ldots, u_{n}$. For a vector $u \in \mathbb{R}^{m} \backslash\{0\}$, define $\gamma_{u}$ as the positive number such that the scaled vector $\gamma_{u} u$ is a boundary point of $\mathcal{G}$. Note that $\gamma_{u}$ is uniquely defined and $\gamma_{u} \geq 1$ for any $u \in \mathcal{G}$. Define also

$$
\beta^{*}=\min _{u \in \mathcal{U}} \frac{1}{\gamma_{u}^{2}}
$$

Theorem 2. For any model (1), we have

(i) $\beta^{*} \leq d(u, \xi)$ for any $u \in \mathcal{U}$ and any $\xi$;

(ii) $0 \leq \beta^{*} \leq 1$;

(iii) $\beta^{*}=1$ if and only if all $u_{i} \in \mathcal{U}$ belong to the boundary of the Elfving set for $\mathcal{U}$. 
Proof of Theorem 2. For any $u \in \mathbb{R}^{m} \backslash\{0\}$, let $\xi_{u}^{*}$ be a $c$-optimal design (for the vector $c=u$ ). Then it follows from the optimality of the design $\xi_{u}^{*}$ that for any $u \in \mathcal{U}$ and any design $\xi$

$$
d(u, \xi)=u^{T} M^{-}(\xi) u \geq u^{T} M^{-}\left(\xi_{u}^{*}\right) u,
$$

where $A^{-}$denotes a generalized inverse of the matrix $A$. Moreover, the right hand side of this inequality is known (Pukelsheim (1993)) to be

$$
u^{T} M^{-}\left(\xi_{u}^{*}\right) u=\frac{1}{\gamma_{u}^{2}}
$$

This proves the assertion (i). Assertion (ii) follows from the definition of $\beta^{*}$ and the fact that $\gamma_{u} \geq 1$ for any $u \in \mathcal{U}$. For a given $u \in \mathcal{U}, \gamma_{u}=1$ if $u$ is a boundary point of the Elfving set $\mathcal{G}$. Therefore, $\beta^{*}=1$ if and only if all $u_{i} \in \mathcal{U}$ are boundary points of $\mathcal{G}$, which proves (iii).

Remark 5 (model with intercept). For models with a constant term we always have $\beta^{*}=1$ as all points $u_{i}=\left(1, v_{i}^{T}\right)^{T}$ are located at the boundary of the corresponding Elfving set.

\section{Numerical comparisons}

In this section, we present a few numerical comparisons of the algorithms. We discuss the performance of the algorithms for several polynomial, exponential and rational regression models. To be precise, we consider the regression model

$$
Y=\theta^{T} f(x)+\varepsilon,
$$

where $f(x)=\left(f_{0}(x), \ldots f_{m-1}(x)\right)^{T}$ is the vector of regression functions and the explanatory variable $x$ varies in a finite set, say $\mathcal{X}=\left\{x_{1}, \ldots, x_{n}\right\}$. In this case, we have $\mathcal{U}=\left\{f\left(x_{1}\right), \ldots, f\left(x_{n}\right)\right\}$ and the regression model can be written in the form (1) with $u=f(x)$. For the polynomial regression model we choose

$$
f(x)=\left(1, x, \ldots, x^{m-1}\right)^{T}, \quad m=3,4,5,6,
$$

while the exponential and rational models are given by

$$
\begin{aligned}
& f(x)=\left(1, e^{-x}, x e^{-x}\right)^{T}, \\
& f(x)=\left(e^{-x}, x e^{-x}, e^{-2 x}, x e^{-2 x}\right)^{T}, \\
& f(x)=\left(1, e^{-x}, x e^{-x}, e^{-2 x}, x e^{-2 x}\right)^{T}, \\
& f(x)=\left(1,1 /(1+x), 1 /(1+x)^{2}\right)^{T} .
\end{aligned}
$$

We consider two design spaces that correspond to Table 1 and Table 2, respectively. Specifically, we consider

$$
\mathcal{X}_{1}=\{4 i / 19 \mid i=0, \ldots, 19\} \quad \text { and } \quad \mathcal{X}_{2}=\{4 i / 39 \mid i=0, \ldots, 39\}
$$


Table 1: The number of iterations required to achieve precision (9) by algorithm (2) with parameters $\beta_{r}=\gamma \beta^{(r)}$ and by Titterington's algorithm with $\beta_{r}=1$. The initial design $\xi^{(0)}$ is a uniform distribution on the set $\mathcal{X}_{1}=\{4 i / 19 \mid i=0, \ldots, 19\}$.

\begin{tabular}{|l|ccccccc|cc||c|}
\hline & \multicolumn{8}{|c||}{ Algorithm $(2)$ with $\beta_{r}=\gamma \beta^{(r)}$} & $\beta_{r}=1$ \\
\hline$\gamma$ & 0 & 0.1 & 0.2 & 0.3 & 0.4 & 0.5 & 0.6 & 0.7 & 0.8 & \\
\hline$\left(1, x, x^{2}\right)$ & 104 & 97 & 91 & 84 & 78 & 71 & 65 & 58 & 69 \\
$\left(1, x, x^{2}, x^{3}\right)$ & 130 & 121 & 113 & 104 & 96 & 88 & 79 & 71 & 98 \\
$\left(1, x, \ldots, x^{4}\right)$ & 82 & 77 & 72 & 67 & 61 & 56 & 51 & 45 & 66 \\
$\left(1, x, \ldots, x^{5}\right)$ & 96 & 89 & 82 & 75 & 68 & 61 & 53 & & 80 \\
$\left(1, e^{-x}, x e^{-x}\right)$ & 131 & 123 & 115 & 108 & 100 & 92 & 84 & 76 & 90 \\
$\left(1,1 /(1+x), 1 /(1+x)^{2}\right)$ & 105 & 98 & 92 & 85 & 79 & 73 & 66 & 60 & 71 \\
$\left(e^{-x}, x e^{-x}, e^{-2 x}, x e^{-2 x}\right)$ & 221 & 208 & 196 & 183 & 170 & 158 & 145 & 133 & 167 \\
$\left(1, e^{-x}, x e^{-x}, e^{-2 x}, x e^{-2 x}\right)$ & 136 & 127 & 118 & 109 & 100 & 91 & 83 & 74 & 109 \\
\hline
\end{tabular}

In all cases a uniform distribution on the design space $\mathcal{X}$ was used as starting design $\xi^{(0)}$, and the iterations of the algorithm (2) were performed until the precision

$$
\max _{i=1, \ldots, n} d\left(u_{i}, \xi^{(r)}\right) \leq 1.001 m
$$

was reached. In Table 1 and 2 we present the number of iterations required to reach this precision by algorithm (2) with $\beta_{r}=\gamma \beta^{(r)}$, for various values of $\gamma$. The tables also contain the results for Titterington's algorithm, which uses constant parameter $\beta_{r}=1$ (see the right columns in the tables). The empty space in the table indicates that the corresponding algorithm did not converge to the optimal design.

Both tables show very similar results. The performance of the algorithm (2) is improved if larger values are used for the parameter $\gamma$. The worst case corresponds to the choice $\beta_{r}=0$, and this is improved by all other methods. If $\gamma$ is small, the algorithm with $\beta_{r}=1$ is still better than the method proposed in this paper. However this picture is changing if values $\gamma \geq 0.5$ are used in the procedure. Note that it follows from the proof of Theorem 1 that the sequence of determinants generated by the algorithm (2) may still be nondecreasing for values of $\beta_{r}$, which are slightly larger than $\beta^{(r)} / 2$ and for this reason our numerical comparison also includes the cases $\gamma=0.6,0.7$ and 0.8 (see Tables 1 and 2). If $\gamma \geq 0.5$, the algorithm (2) is at least comparable to the case $\beta_{r}=1$, and in many cases it yields a substantially smaller number of iterations for achieving the desired precision. 
Table 2: The number of iterations required to achieve precision (9) by algorithm (2) with parameters $\beta_{r}=\gamma \beta^{(r)}$ and by Titterington's algorithm with $\beta_{r}=1$. The initial design $\xi^{(0)}$ is a uniform distribution on the set $\mathcal{X}_{2}=\{4 i / 39 \mid i=0, \ldots, 39\}$.

\begin{tabular}{|l|cccccc|ccc||c|}
\hline & \multicolumn{8}{|c||}{ Algorithm $(2)$ with $\beta_{r}=\gamma \beta^{(r)}$} & $\beta_{r}=1$ \\
\hline$\gamma$ & 0 & 0.1 & 0.2 & 0.3 & 0.4 & 0.5 & 0.6 & 0.7 & 0.8 & \\
\hline$\left(1, x, x^{2}\right)$ & 250 & 235 & 219 & 204 & 188 & 172 & 157 & 141 & 167 \\
$\left(1, x, x^{2}, x^{3}\right)$ & 329 & 308 & 287 & 266 & 244 & 223 & 202 & 181 & 247 \\
$\left(1, x, \ldots, x^{4}\right)$ & 235 & 219 & 204 & 188 & 173 & 157 & 142 & 127 & 188 \\
$\left(1, x, \ldots, x^{5}\right)$ & 281 & 262 & 244 & 226 & 207 & 189 & 170 & 152 & 234 \\
$\left(1, e^{-x}, x e^{-x}\right)$ & 294 & 276 & 258 & 239 & 221 & 202 & 184 & 166 & 197 \\
$\left(1,1 /(1+x), 1 /(1+x)^{2}\right)$ & 136 & 128 & 120 & 111 & 103 & 94 & 86 & 77 & 91 \\
$\left(e^{-x}, x e^{-x}, e^{-2 x}, x e^{-2 x}\right)$ & 404 & 382 & 359 & 337 & 314 & 291 & 269 & 246 & 224 & 304 \\
$\left(1, e^{-x}, x e^{-x}, e^{-2 x}, x e^{-2 x}\right)$ & 213 & 199 & 185 & 171 & 157 & 143 & 130 & 116 & 171 \\
\hline
\end{tabular}

\section{$5 \quad$ Other optimality criteria}

\subsection{Bayesian D-optimal designs}

In this section we briefly discuss an extension of the multiplicative algorithm to the Bayesian $D$-optimal design problem. To be precise, consider the non-linear regression model

$$
Y=\eta(x, \theta)+\varepsilon,
$$

where $\eta$ is a known function, $\theta \in \Theta \subset \mathbb{R}^{m}$ denotes the unknown parameter and the explanatory variable $x$ varies in the finite design space $\mathcal{X}=\left\{x_{1}, \ldots, x_{n}\right\}$. Under the assumption of a normally distributed homoscedastic error it was shown by Jennrich (1969) that asymptotically the covariance matrix of the least squares estimate for the parameter $\theta$ is proportional to the matrix $M^{-1}(\xi, \theta)$, where $\xi$ is the given design,

$$
M(\xi, \theta)=\int_{\mathcal{X}} f(x, \theta) f^{T}(x, \theta) d \xi(x),
$$

and $f(x, \theta)=\frac{\partial}{\partial \theta} \eta(x, \theta)$ is the gradient of $\eta$ with respect to $\theta$. A Bayesian $D$-optimal design maximizes

$$
\Phi_{\pi}(\xi)=\int_{\Theta} \log \operatorname{det} M(\xi, \theta) \pi(d \theta)
$$

where $\pi$ denotes a given prior distribution on the parameter space $\Theta$ [see e.g. Chaloner and Larntz (1989) or Chaloner and Verdinelli (1996)]. Define

$$
d_{\pi}(x, \xi)=\int_{\Theta} f^{T}(x, \theta) M^{-1}(\xi, \theta) f(x, \theta) \pi(d \theta)
$$


and note that a design $\xi^{*}$ is Bayesian $D$-optimal if and only if the inequality

$$
d_{\pi}\left(x, \xi^{*}\right) \leq m
$$

holds for all $x \in \mathcal{X}$. We consider the multiplicative algorithm

$$
w_{i}^{(r+1)}=w_{i}^{(r)} \frac{d_{\pi}\left(x_{i}, \xi^{(r)}\right)-\beta_{r}}{m-\beta_{r}}, \quad i=1, \ldots, n,
$$

where the procedure is terminated if

$$
\max _{i=1, \ldots, n} d_{\pi}\left(x_{i}, \xi^{(r)}\right) \leq 1.001 m
$$

Some numerical results for the algorithm (10) are given in Table 3. We can see that the performance of algorithm (10) is similar to the performance of algorithm (2).

Table 3: The number of iterations required to achieve precision (11) by algorithm (10) with parameters $\beta_{r}=\gamma \beta^{(r)}$ and distribution $\pi=$

\begin{tabular}{|c|c|c|c|c|c|c|c|c|c|}
\hline & \multicolumn{8}{|c|}{ Algorithm (10) with $\beta_{r}=\gamma \beta^{(r)}$} & $\beta_{r}=1$ \\
\hline$\gamma$ & 0 & 0.1 & 0.2 & 0.3 & 0.4 & 0.5 & $0.6 \quad 0$ & 0.7 & \\
\hline$\left.\overline{\theta x}, x e^{-\theta x}\right)$ & 178 & 167 & 156 & 145 & 133 & 122 & 1111 & 100 & 120 \\
\hline$\left.(\theta+x), 1 /(\theta+x)^{2}\right)$ & 147 & 138 & 129 & 120 & 110 & 101 & & 83 & 98 \\
\hline$\left(e^{-\theta x}, x e^{-\theta x}, e^{-2 x}, x e^{-2 x}\right)$ & 322 & 296 & 270 & 244 & 218 & 192 & 165 & & 242 \\
\hline$\left(1, e^{-\theta x}, x e^{-\theta x}, e^{-2 x}, x e^{-2 x}\right)$ & 101 & 95 & 88 & 81 & 75 & 68 & 61 & 55 & 81 \\
\hline
\end{tabular}
$\{0.7,0.8,0.9,1,1.1,1.2,1.3 ; 1 / 7, \ldots, 1 / 7\}$. The initial design $\xi^{(0)}$ is a uniform distribution on the set $\mathcal{X}_{3}=\{3 i / 19 \mid i=0, \ldots, 19\}$.

Based on extensive numerical calculations we conjecture that Theorem 1 can be extended from the $D$-optimality criterion to the Bayesian $D$-optimality criterion; that is, the sequence of designs defined by (10) with $\beta_{r} \leq \frac{1}{2} \min _{i=1, \ldots, n} d_{\pi}\left(x_{i}, \xi^{(r)}\right)$ or $\beta_{r}=1$ yields a nondecreasing sequence $\left\{\Phi_{\pi}\left(\xi^{(r)}\right)\right\}$.

\section{$5.2 \quad A-, E$ - and $c$-optimal designs}

Let us finally discuss an extension of the multiplicative algorithm to the $A-, E$ - and $c$-optimal design problems. Consider a general (differentiable) optimality criterion $\Phi$ such that $\Phi$-optimal design either maximizes $\Phi(M(\xi))$ or minimizes $\Psi\left(M^{-1}(\xi)\right)$. The nonnegative function $d\left(u_{i}, \xi\right)$ is then generalised either to

$$
\phi\left(u_{i}, \xi\right)=\frac{\partial \Phi(M(\xi))}{\partial w_{i}}=u_{i}^{T} \stackrel{\circ}{\Phi}(\xi) u_{i}
$$

or

$$
\phi\left(u_{i}, \xi\right)=-\frac{\partial \Psi\left(M^{-1}(\xi)\right)}{\partial w_{i}}=u_{i}^{T} M^{-1}(\xi) \stackrel{\circ}{\Psi}(\xi) M^{-1}(\xi) u_{i}
$$


where

$$
\stackrel{\circ}{\Phi}(\xi)=\left.\frac{\partial \Phi(M)}{\partial M}\right|_{M=M(\xi)}, \stackrel{\circ}{\Psi}(\xi)=\left.\frac{\partial \Psi\left(M^{-1}\right)}{\partial M^{-1}}\right|_{M=M(\xi)} .
$$

For the $A$-optimality criterion $\Psi\left(M^{-1}(\xi)\right)=\operatorname{tr}\left(M^{-1}(\xi)\right)$, it follows $\stackrel{\circ}{\Psi}(\xi)=I_{m}$ and the function $\phi$ has the form

$$
\phi_{A}\left(u_{i}, \xi\right)=u_{i}^{T} M^{-2}(\xi) u_{i}
$$

If the multiplicity of the minimal eigenvalue of the matrix $M(\xi)$ equals 1 , then for the $E$-optimality criterion $\Phi(M(\xi))=\lambda_{\min }(M(\xi)$ ) (which is in general not differentiable) the function $\phi$ has the form

$$
\phi_{E}\left(u_{i}, \xi\right)=\left(p^{T} u_{i}\right)^{2},
$$

where $p$ is a normalized eigenvector corresponding to the minimal eigenvalue of $M(\xi)$. If the matrix $M(\xi)$ has rank $m$, the function $\phi$ for the $c$-optimality criterion $\Psi\left(M^{-1}(\xi)\right)=$ $c^{T} M^{-1}(\xi) c$ is given by

$$
\phi_{c}\left(u_{i}, \xi\right)=\left(c^{T} M^{-1}(\xi) u_{i}\right)^{2} .
$$

For $D^{-}, A-, E$ - and coptimality we consider an algorithm in the following form

$$
w_{i}^{(r+1)}=w_{i}^{(r)} \frac{\phi\left(u_{i}, \xi^{(r)}\right)+\beta_{r}}{b\left(\xi^{(r)}\right)+\beta_{r}}, \beta_{r} \in \mathbb{R}
$$

where $b(\xi)=\operatorname{tr} M(\xi) \stackrel{\circ}{\Phi}(\xi)$ or $b(\xi)=\operatorname{tr} M^{-1}(\xi) \stackrel{\circ}{\Psi}(\xi)$. Note that sum of weights at the next iteration equals 1 as

$$
b\left(\xi^{(r)}\right)=\sum_{j} w_{j}^{(r)} \phi\left(u_{j}, \xi^{(r)}\right)
$$

and that for the $D$-criterion (12) reduces to the recursive relation defined in (2).

Numerical calculations show that the algorithm (12) with $\beta_{r}=0$ is generally not monotonic (that is, the sequence $\left\{\Phi\left(M\left(\xi^{(r)}\right)\right\}\right.$ is not a monotone sequence) for the $A$-, $E$ - and $c$-criteria, in contrast to the case of $D$-optimality. We therefore need to use positive values of $\beta_{r}$ in the algorithm (12). We conjecture that for $A-, E$ - and $c$ optimality, the sequence of designs $\left\{\xi^{(r)}\right\}$ obtained by the recurrent formula (12) with $\beta_{r} \geq \frac{1}{2} b\left(\xi^{(r)}\right)$ yields a monotonic sequence $\left\{\Phi\left(\xi^{(r)}\right)\right\}$.

In numerical studies, we use $\beta_{r}=(1-\gamma) b\left(\xi^{(r)}\right)$ for different values of $\gamma, 0 \leq \gamma<1$. We run the iterations of the algorithm (12) until the precision

$$
\max _{i=1, \ldots, n} \phi\left(u_{i}, \xi^{(r)}\right) \leq 1.001 b\left(\xi^{(r)}\right)
$$

is achieved. Some results are given in Tables 4 and 5 and demonstrates that multiplicative algorithms can also be applied in to other optimality criteria.

Acknowledgements. The work was partly supported by the Deutsche Forschungsgemeinschaft (SFB 475: Komplexitätsreduktion in multivariaten Datenstrukturen, Teilprojekt A2). The work of H. Dette was also supported in part by a NIH grant award 
Table 4: The number of iterations required to achieve precision (13) by algorithm (12) for E-optimality with parameters $\beta_{r}=(1-\gamma) b\left(\xi^{(r)}\right)$. The initial design $\xi^{(0)}$ is a uniform distribution on the set $\mathcal{X}_{3}=\{3 i / 19 \mid i=0, \ldots, 19\}$.

\begin{tabular}{|l|cccccc|cccc|}
\hline & \multicolumn{1}{|c|}{ Algorithm $(12)$ with $\beta_{r}=(1-\gamma) b\left(\xi^{(r)}\right)$} \\
\hline$\gamma$ & 0 & 0.1 & 0.2 & 0.3 & 0.4 & 0.5 & 0.6 & 0.7 & 0.8 & 0.9 \\
\hline$\left(1, x, x^{2}\right)$ & 100 & 95 & 90 & 85 & 80 & 75 & 70 & 65 & & \\
$\left(1, x, x^{2}, x^{3}\right)$ & 129 & 122 & 116 & 110 & 103 & 97 & 90 & 84 & 78 & \\
$\left(1, x, \ldots, x^{4}\right)$ & 51 & 48 & 46 & 43 & 41 & 38 & 35 & 33 & 31 & \\
$\left(1, x, \ldots, x^{5}\right)$ & 215 & 204 & 194 & 183 & 172 & 162 & 151 & 141 & 130 & 120 \\
$\left(1, e^{-x}, x e^{-x}\right)$ & 265 & 252 & 239 & 226 & 213 & 200 & 187 & 174 & & \\
$\left(1,1 /(1+x), 1 /(1+x)^{2}\right)$ & 115 & 109 & 103 & 98 & 92 & 86 & 80 & 75 & 69 & 62 \\
$\left(e^{-x}, x e^{-x}, e^{-2 x}, x e^{-2 x}\right)$ & 493 & 469 & 444 & 419 & 395 & 370 & 346 & 321 & 297 & 272 \\
$\left(1, e^{-x}, x e^{-x}, e^{-2 x}, x e^{-2 x}\right)$ & 90 & 86 & 81 & 77 & 72 & 68 & 63 & 59 & 54 & 50 \\
\hline
\end{tabular}

Table 5: The number of iterations required to achieve precision (13) by algorithm (12) for A-optimality with parameters $\beta_{r}=(1-\gamma) b\left(\xi^{(r)}\right)$. The initial design $\xi^{(0)}$ is a uniform distribution on the set $\mathcal{X}_{3}=\{3 i / 19 \mid i=0, \ldots, 19\}$.

\begin{tabular}{|l|cccccc|cccc|}
\hline & \multicolumn{8}{|c|}{ Algorithm $(12)$ with $\beta_{r}=(1-\gamma) b\left(\xi^{(r)}\right)$} \\
\hline$\gamma$ & 0 & 0.1 & 0.2 & 0.3 & 0.4 & 0.5 & 0.6 & 0.7 & 0.8 & 0.9 \\
\hline$\left(1, x, x^{2}\right)$ & 270 & 257 & 244 & 230 & 217 & 204 & 190 & 177 & 164 & 151 \\
$\left(1, x, x^{2}, x^{3}\right)$ & 126 & 120 & 114 & 107 & 101 & 94 & 88 & 82 & 75 & 69 \\
$\left(1, x, \ldots, x^{4}\right)$ & 330 & 314 & 298 & 282 & 266 & 249 & 233 & 217 & 202 & 187 \\
$\left(1, x, \ldots, x^{5}\right)$ & 270 & 256 & 243 & 229 & 215 & 201 & 187 & 173 & 159 & 143 \\
$\left(1, e^{-x}, x e^{-x}\right)$ & 229 & 218 & 207 & 195 & 184 & 173 & 161 & 150 & 139 & 128 \\
$\left(1,1 /(1+x), 1 /(1+x)^{2}\right)$ & 116 & 110 & 104 & 99 & 93 & 87 & 81 & 75 & 70 & 63 \\
$\left(e^{-x}, x e^{-x}, e^{-2 x}, x e^{-2 x}\right)$ & 520 & 494 & 468 & 442 & 416 & 391 & 365 & 339 & 313 & 287 \\
$\left(1, e^{-x}, x e^{-x}, e^{-2 x}, x e^{-2 x}\right)$ & 90 & 85 & 81 & 76 & 72 & 68 & 63 & 59 & 54 & 49 \\
\hline
\end{tabular}

IR01GM072876:01A1. The authors are very grateful to Radoslav Harman who carefully checked the proofs of the main result and made a few very useful comments. The authors are also grateful to M. Stein who typed parts of this paper with considerable technical expertise.

\section{References}

K. Chaloner and K. Larntz. Optimal Bayesian design applied to logistic regression experiments. J. Statist. Plann. Inference, 21(2):191-208, 1989. 
K. Chaloner and I. Verdinelli. Bayesian experimental design: a review. Statist. Sci., 10(3):273-304, 1995.

G. Elfving. Optimum allocation in linear regression theory. Ann. Math. Statistics, 23: 255-262, 1952.

V. V. Fedorov. Theory of optimal experiments. Academic Press, New York, 1972.

F. R. Gantmacher. The Theory of Matrices, volume 1. Chelsea, New York, 1959.

R. Harman and L. Pronzato. Improvements on removing nonoptimal support points in D-optimum design algorithms. Stat. \& Prob. Letters, 77(1):90-94, 2007.

R. I. Jennrich. Asymptotic properties of non-linear least squares estimators. Ann. Math. Statist., 40:633-643, 1969.

J. Kiefer. General equivalence theory for optimum designs (approximate theory). The Annals of Statistics, 2(5):849-879, 1974.

J. Kiefer and J. Wolfowitz. The equivalence of two extremum problems. Canad. J. Math., 12:363-366, 1960.

S. Mandal and B. Torsney. Construction of optimal designs using a clustering approach. J. Statist. Plann. Inference, 136:1120-1134, 2006.

A. Pázman. Foundations of optimum experimental design. Reidel, Dordrecht, 1986.

L. Pronzato. Removing non-optimal support points in $D$-optimum design algorithms. Statist. Probab. Lett., 63(3):223-228, 2003.

L. Pronzato, H. P. Wynn, and A. A. Zhigljavsky. Dynamical search. Chapman \& Hall/CRC, Boca Raton, FL, 2000.

F. Pukelsheim. Optimal design of experiments. John Wiley \& Sons Inc., New York, 1993.

F. Pukelsheim and S. Rieder. Efficient rounding of approximate designs. Biometrika, 79(4):763-770, 1992.

F. Pukelsheim and B. Torsney. Optimal weights for experimental designs on linearly independent support points. Ann. Statist., 19(3):1614-1625, 1991.

S. D. Silvey. Optimal design. Chapman \& Hall, London, 1980.

S. D. Silvey, D. M. Titterington, and B. Torsney. An algorithm for optimal designs on a finite design space. Comm. Statist., Theory Meth., 14:1379-1389, 1978.

D. M. Titterington. Optimal design: some geometrical aspects of D-optimality. Biometrika, 62(2):313-320, 1975. 
D. M. Titterington. Algorithms for computing D-optimal design on finite design spaces. In Proc. of the 1976 Conf. on Information Science and Systems, John Hopkins University, (3):213-216, 1976.

D. M. Titterington. Estimation of correlation coefficients by ellipsoidal trimming. Applied Statistics, 27(3):227-234, 1978.

B. Torsney. W-iterations and ripples therefrom. In Optimal Design and Related Areas in Optimization and Statistics (eds. L.Pronzato and A.Zhigljavsky), pages 1-12. Springer-Verlag, N.Y., 2007.

B. Torsney and S. Mandal. Construction of constrained optimal designs. In Optimum design 2000 (eds. A.C. Atkinson, B. Bogacka and A.Zhigljavsky), pages 141-152. Kluwer Acad. Publ., Dordrecht, 2001.

H. P. Wynn. Results in the theory and construction of $D$-optimum experimental designs. J. Roy. Statist. Soc. Ser. B, 34:133-147, 1972. 